\title{
An agent-based model of innovation diffusion: network structure and coexistence under different information regimes
}

\author{
Giovanni Pegoretti · Francesco Rentocchini · \\ Giuseppe Vittucci Marzetti
}

November 12, 2011

\begin{abstract}
The paper analyzes how the structure of social networks affects innovation diffusion and competition under different information regimes. Diffusion is modeled as the result of idiosyncratic adoption thresholds, local network effects and information diffusion (broadcasting and demonstration effect from previous adopters). A high social cohesion decreases the probability of one innovation cornering the market. Nonetheless, with imperfect information, in small-world networks the higher speed of diffusion produced by the low average distance increases this probability. A low social cohesion also increases the probability of falling into traps of under-adoption. However, such probability is significantly lower with imperfect information, because such regime is characterized by higher levels of market concentrations and this reduces the frictions due to the coexistence of non-compatible product innovations.
\end{abstract}

Keywords Agent-based model · Innovation diffusion · Network effects · Social networks · Small-world

JEL Classification D830 D850 M310 O330.

The three authors contributed equally to the paper and share Sections 1 and 5. Still, Section 2 could be attributed to Francesco Rentocchini, while Sections 3 and 4 to Giuseppe Vittucci Marzetti.

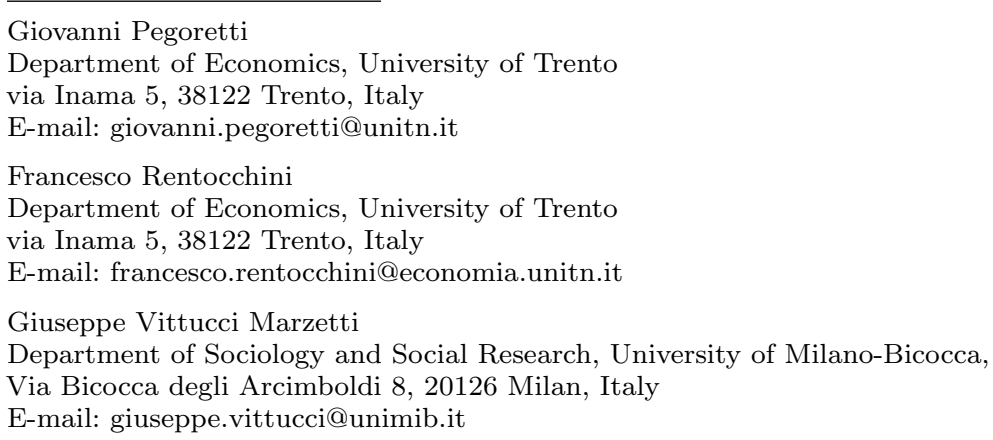




\section{Introduction}

Social and economic networks are the subject of a fast growing literature, ranging from the analysis of learning processes, consensus formation (e.g. Golub and Jackson 2010) and risk-sharing (e.g. Bramoullé and Kranton 2007), to the analysis of industrial clusters (e.g. Morrison 2008) and R\&D collaborations among firms (e.g. Goyal and Moraga-Gonzalez 2001). ${ }^{1}$

In the economics of innovation, network analysis has been fruitfully applied to study the processes of knowledge creation and diffusion (Cowan and Jonard 2003, 2004), and the patterns of innovation adoption (e.g. Morris 2000; Delre et al. 2007a; López-Pintado 2008). As for the latter, along with a number of works that models innovation diffusion as a stochastic process of "percolation" in a grid (Frenken et al. 2008; Hohnisch et al. 2008; Delre et al. 2010), thus assuming regular networks, economists have also started analyzing the effects of the different network structures (Abrahamson and Rosenkopf 1997; Arenas et al. 2002; Lee et al. 2006; Choi et al. 2010).

Our contribution follows this last stream. We study a model of innovation diffusion with many incompatible products competing for adopters, mainly focusing on demand-side dynamics. In particular, we extend the model analyzed in Lee et al. (2006) by considering more than two product innovations and distinguishing the case of perfect information from that of imperfect information. In the former, all potential adopters are perfectly informed about the existence of the different innovations. Each agent has got an idiosyncratic willingness to adopt and she is influenced by her neighbors' choices, because of the existence of local network externalities. On the contrary, with imperfect information agents are not always informed about the existence of all the products. This information diffuses through the network via a demonstration effect (direct contact with adopters) and broadcasting (advertising or marketing) (e.g. Bass 1969; Bemmaor and Lee 2002).

To start with, we analyze how the network structure affects the speed of diffusion of a single product innovation in a population of agents characterized by idiosyncratic individual adoption thresholds under the two information regimes (perfect vs. imperfect information). We show that, with perfect information, the innovation diffuses faster when the network is completely random. On the contrary, with imperfect information, the fastest pace is reached in small-world networks.

Then, we extend the analysis to incorporate the process of competition among alternative innovations. We study how the interplay between network structure and information regime affects the probability of coexistence of the different innovations. Results show that, with imperfect information, winner-take-all solutions (i.e. one innovation cornering the market and displacing completely the others) are more frequent than expected, even in markets characterized by strong social cohesion. Moreover, such outcomes become significantly more frequent when the average distance in the network is low, despite a possible high clustering, as in the case of small-world networks.

We also generalize Choi et al.'s (2010) results about the relation between network structure and penetration rates to the many-products case by showing that a weak cliquishness increases the probability of falling into a trap of under-

1 Thorough and concise surveys are Newman (2003) and Uzzi et al. (2007). Introductory textbooks are Goyal (2007), Vega-Redondo (2007) and Jackson (2008). 
adoption also with more than one product. Moreover, we find that such probability can be significantly lower in case of imperfect information. The reason is that imperfect information leads on average to higher market concentration, and this in turn reduces the frictions due to product coexistence (which leads to a lower level of positive network externalities) and increases the penetration rate.

Our model has straightforward application in fashionable markets, e.g. fashion, electronic devices, software programs and Social Networking Sites (SNS). In such markets, three main characteristics of the competing products easily fit our assumptions: i) they are mainly substitutes; ii) they arrive roughly at the same time; iii) they are characterized by strong network externalities. Some notable real-world examples include the successful marketing campaign "calling circle" launched by MCI, which allowed the company to increase its market share against AT\&T (Lee et al. 2006); the case of Vorwerk in the market for household appliances; the competition between Microsoft, IBM and Sun Microsystems/Oracle in the market for office software suites (Krishnamurthy 2003); the fierce competition that recently characterized social network sites (Boyd and Ellison 2008).

The paper is organized as follows. Section 2 briefly reviews the literature on the role of local network effects in innovation diffusion. Section 3 presents the model. Section 4 discusses the main results. Section 5 concludes with some final remarks.

\section{Theoretical background}

Although the literature on innovation diffusion is vast and heterogeneous, the role played by network topologies in the process has been only recently studied (comprehensive surveys are Karshenas and Stoneman 1995; Geroski 2000; Rogers 2003; Valente 2005). This has allowed to cope with issues related with the effects that neighbors' choices have on adoption decisions. Indeed, it is now widely acknowledged that consumers base their own choices on the information derived from previous adopters, so generating a social bandwagon pressure to conform. When uncertainty is very high, agents tend to ground their decisions on social cues (Abrahamson and Rosenkopf 1997).

Within this stream of research, Delre et al. (2007a) have joined the literature on innovation diffusion - epidemic and threshold models - with the marketing literature on the diffusion of products in fashionable markets (Bass 1969, 2004; Chatterjee and Eliashberg 1990). They consider both agents' preferences and network effects arising from word-of-mouth communication and show that: i) diffusion is faster in small-world networks; ii) agents' heterogeneity increases the speed of diffusion. ${ }^{2}$

Finally, the interplay between network structure, agents' heterogeneity and product penetration rates in markets characterized by local network externalities is analyzed by Choi et al. (2010), who show that the probability of falling into

2 The latter result is analytically proved by Young (2009), who however use a mean-field approximation and deliberately neglect the effects of the network topology. Young (2009) groups diffusion models into three broad classes: i) contagion, where agents adopt when they come in contact with other adopters; ii) social influence, where people adopt when enough other people in the group have adopted; iii) social learning, where people adopt once they see enough empirical evidence to convince them that the innovation is worth adopting. 
under-adoption traps is higher in random networks, because of the low clustering, that could reinforce local positive externalities. ${ }^{3}$

Albeit important in showing the role played by network structure on diffusion patterns, these contributions does not analyze the process of diffusion of many incompatible innovations, competing for the same user base; and, in particular, the effect of the structure of social relations among consumers on the probability of outcomes with standardization (winner-take-all solutions) vs. coexistence of the different products in the market.

This issue is addressed by Lee et al. (2006), who investigate the role of the structure of social networks when two incompatible innovations compete for the same population of adopters. The authors find that, in networks characterized by strong social cohesion (high clustering), the usual outcome is one of coexistence, even when there are significant differences in the installed bases; and conclude that emphasizing the role played by global network effects, while ignoring local ones, necessarily produces a bias towards winner-take-all outcomes. ${ }^{4}$

In what follows, we extend Lee et al.'s (2006) analysis by considering more than two innovations and two alternative information regimes-perfect vs. imperfect information.

\section{The model}

Let $\mathcal{N}=\{1,2, \ldots, N\}$ be the set of agents. Each agent is a node in an undirected binary network $\Gamma=(\mathcal{N}, \mathcal{G})$, where $\mathcal{G}$ is the set of links. Let $\mathcal{N}_{i}$ be the set of neighbors of agent $i$ :

$$
\mathcal{N}_{i}=\{j \in \mathcal{N} \backslash\{i\}:(i, j) \in \mathcal{G}\}
$$

$\mathcal{X}=\{1,2, \ldots, X\}$ is the set of available product innovations and $\mathbf{a}(t) \in(\{0\} \cup \mathcal{X})^{N}$ the vector of agents' actions, whose generic element $a_{i}(t)$ is the adoption choice of agent $i$ at time $t$ and $a_{i}(t)=0$ if the agent decides not to adopt any innovation.

Adoption dynamics are driven by the myopic maximization of individual consumer surplus. The surplus of agent $i$ is:

$$
\pi_{i}(t)= \begin{cases}0 & \text { if } a_{i}(t)=0 \\ r_{i}+\alpha \frac{\left|\left\{j \in \mathcal{N}_{i}: a_{j}(t-1)=a_{i}(t)\right\}\right|}{\left|\mathcal{N}_{i}\right|} & \text { otherwise }\end{cases}
$$

where $r_{i}=p_{i}^{m}-p$ is the difference between the basic willingness-to-pay of agent $i$ for any innovation and the price charged for it, and $\alpha$ is an exogenous parameter supposed to measure the strength of network effects.

In words, the surplus generated by the adoption of a certain innovation for an agent is a function of: i) her idiosyncratic willingness-to-pay for any innovation

\footnotetext{
3 According to their definition, the system falls into a trap of under-adoption when the adoption process stops before it reaches $16 \%$ of the population, i.e., the proportion of innovators and early adopters according to Rogers's (2003) categorization.

4 Uchida and Shirayama (2008) show that coexistence can result also in networks characterized by positive assortativity, i.e., positive correlation between node degrees; and coexistence is also the normal outcome in models with local interaction where agents can jointly adopt two or more products (see, for instance Goyal and Janssen 1997; Mahdian et al. 2007).
} 
$\left(p_{i}^{m}\right)$; ii) the price charged for it $(p)$; iii) the fraction of her neighbors who have adopted the same innovation in the previous period. ${ }^{5,6}$

We assume that an agent can adopt only one innovation per period. Moreover, in each period, each agent must reacquire the innovation, thus having the possibility to revise her choice by choosing an alternative innovation without incurring any additional cost. When for a certain agent in a certain period two or more actions give the same surplus, the agent chooses among them uniformly at random.

In order to better focus on the role of local network externalities, we assume a constant and equal price and the same intrinsic quality for all the innovations, so abstracting from the effects of supply-side factors, like economies of scale - static (increasing returns to scale) and dynamic (learning-by-doing). ${ }^{7}$ Moreover, we also abstract from the effects of habits and other inertial factors in adoption decisions (e.g. learning-by-using). ${ }^{8}$

Like in Katz and Shapiro (1985) and Lee et al. (2006), among the others, we assume $r_{i}$ is heterogeneous across customers and drawn from a normal distribution with negative mean, thus assuming that the basic willingness-to-pay is on average lower than the price, i.e. agents are on average reluctant to be the first to adopt an innovation. The maximization of $\pi_{i}(t)$ according to Eq. (1) therefore implies the existence of a threshold for the adoption.

In order to analyze the case of imperfect information, we restrict the set of actions available to each agent in each period. Formally, we assume:

$$
\mathbf{a}(t) \in \times_{i \in \mathcal{N}}\left(\{0\} \cup \mathcal{I}_{i}(t)\right)
$$

where $\mathcal{I}_{i}(t) \subseteq \mathcal{X}$ is the information set of agent $i$, i.e., the set of available innovations known by $i$ at time $t$.

We assume further that agents possess infinite memory (if $x \in \mathcal{I}_{i}(t)$ then $x \in \mathcal{I}_{i}\left(t^{\prime}\right)$, for all $\left.t^{\prime} \geq t\right)$ and no prior information $\left(\mathcal{I}_{i}(0)=\emptyset\right.$ for all $\left.i \in \mathcal{N}\right)$.

5 The assumption of an additive function is quite standard in the literature to model both global (e.g. Katz and Shapiro 1985; Arthur 1989) and local network effects (e.g. Janssen and Jager 2003; Lee et al. 2006; Delre et al. 2007a; Banerji and Dutta 2009; Choi et al. 2010) in the diffusion of products, innovations and technologies. Swann (2002) shows that the utility of the representative user is linearly related to the total network of users if all users have on average the same number of links.

6 Our formulation in terms of consumer surplus differs slightly from Lee et al. (2006), who instead refer generically to payoff and utility gains. Ours is fully consistent with Katz and Shapiro's (1985) original formulation and it seems more in line with the partial equilibrium perspective of the model. The reference to the actions of neighbors in the previous periods is needed to avoid deadlock errors in the simulations.

7 Assuming economies (diseconomies) of scale in the model necessarily leads to a decrease (increase) in the relative price of the innovation which ends up with the largest installed base, and this in turn increases (decreases) ceteris paribus the probability of winner-take-all solutions, no matter the network structure.

8 By introducing frictions in the form of switching costs, all these factors necessarily increase the probability of coexistence, no matter the actual structure of the network. A simple way to introduce them in our model is by adding the agent $i$ to her own neighbors in Eq. (1) in the calculus of the total network effect. One could envisage more complex formulations (e.g. the introduction of a positive term to the function which traces the total periods of use of each innovation and their temporal structure, so to apply some kind of depreciation). No matter the formulation, the effect will always be ceteris paribus an increase in the probability of coexistence. Needless to say, in spite of the fact that we abstract from them in our model, the role of habits and learning-by-using in consumption is probably one of the main reasons why winner-take-all solutions are rarely observed in reality. 
Information on innovation availability diffuses through the network via broadcasting and demonstration effect by previous adopters. In particular, we assume that in each period there is a probability $p$ that $x$ is added to $\mathcal{I}_{i}(t)$, where $p$ depends on the intensity of the diffusion from the central source. ${ }^{9}$ In addition, $x \in \mathcal{I}_{i}(t)$ whenever there is at least one adopter of $x$ among the neighbors of agent $i$, i.e., formally $\exists j \in \mathcal{N}_{i}: a_{j}(t-1)=x$.

To analyze the interaction between network structure and diffusion dynamics, we resort to Watts and Strogatz's (1998) model, which starts from a one-dimensional ring lattice of $n$ nodes with degree $k$, thus assuming that each of $n$ customers maintains $k$ relationships with the others. In this regular network, agents are highly clustered and the average distance among nodes is high. To build other kinds of networks starting from this, one can then rewire each of the links by making random connections between nodes with a certain probability $\beta$. When $\beta$ is rather small (ranging from 0.01 to 0.1 ), the high clustering is preserved while the few shortcuts significantly decrease the average distance (so-called small-world networks). Finally, for $\beta=1$ the network becomes completely random and therefore it exhibits small clustering coefficient and small average distance.

\section{Results}

We implement the model as an agent-based model and present the main results of simulations. ${ }^{10}$ In particular, we analyze in Section 4.1 the diffusion patterns of one single innovation; in Section 4.2 the case of five competing innovations, studying how the probability of coexistence of the different innovations in the market changes by changing the structure of the network and the hypothesis on the information regime.

All the simulations are made starting from a regular network of 1000 agents with degree 10 each $(k=10)$ and analyzing 21 different configurations with $\beta$ ranging from 0.0001 to 1 , equally spaced on a logarithmic scale. Moreover, we assume that $r_{i}$ is normally distributed with mean $\mu=-100$ and standard deviation $\sigma=50$, while the strength of network effects $(\alpha)$ is 500. Finally, as for the case of imperfect information, the broadcasting probability $p$ is kept low and constant, and set equal to $0.001 .^{11}$ Each set of simulations is averaged across 1000 runs.

Finally, in Section 4.3 we analyze the results for alternative combinations of the parameters of the model, namely the distribution of the idiosyncratic adoption threshold $\left(r_{i}\right)$ and the strength of network effects $(\alpha)$, to study the relation between information regimes, network structures, agent heterogeneity and market penetration rates of products.

\footnotetext{
9 One can in principle assume that $p$ is different for each innovation, depending on the marketing efforts of the firm. For an analysis of the effects of the interaction between network structure and different marketing strategies on diffusion in the one innovation case, see Delre et al. (2007b).

10 The model has been implemented in the Laboratory for Simulation Development (LSD)http://www. labsimdev.org. Code available at request.

11 The higher $p$, the more similar the results are to the case of perfect information.
} 


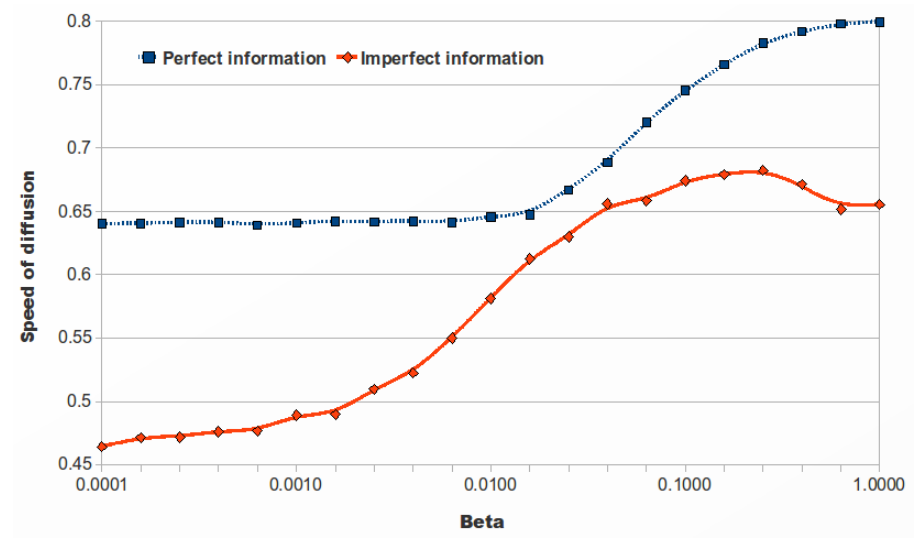

Fig. 1 Average speed of diffusion with perfect (20 steps) and imperfect (200 steps) information

\subsection{Diffusion of a single innovation}

The first result is showed in Figure 1, where the parameter $\beta$ is plotted against the speed of diffusion for a single innovation in the two information regimes. With perfect (imperfect) information, complete adoption is reached on average after 20 (200) time steps. As in Delre et al. (2007a), we calculate the speed of diffusion as:

$$
\rho=\frac{1}{T} \frac{\sum_{t=0}^{T} F(t)}{\sum_{t=0}^{T} f(t)}
$$

where $T$ is the number of time steps, $F(t)$ the cumulative number of adopters at time $t$ and $f(t)$ the number of adopters at $t$.

Apart from the higher speed of diffusion in the case of perfect information, it is worth noting the different effect an increase in $\beta$ produces in the two regimes. With perfect information, the speed increases monotonically from a regular network to a random one, and it starts increasing from $\beta=0.01$. This happens because the clustering coefficient starts decreasing and this reduces the redundancy of some links, thus increasing the extent of the influence of each adoption decision on non adopters. On the contrary, with imperfect information, the speed of diffusion starts increasing earlier, at values of $\beta$ around 0.001 , when the average distance starts falling. This happens because information diffusion mainly relies on the demonstration effect from previous adopters. The decrease in distance makes the few early adopters spread their influence faster in the network. ${ }^{12}$ Moreover, the peak is reached around $\beta=0.2$ and at greater values the speed decreases. This result (which is not an artifact and does not disappear by increasing the number of simulations) is consistent with the one obtained by Delre et al. (2007a), who analyzes the adoption patterns in a similar model. It is due to the fact that, by increasing $\beta$ further, although the average distance keeps on somewhat declining, the simultaneous steep

\footnotetext{
12 While, with perfect information, after one step the average number of early adopters is about $23(N(1-\Phi(-\mu / \sigma)))$, with imperfect information the demonstration effect is at first on average triggered by one adopter only. Indeed, in the latter case the expected number of early adopters informed via broadcasting after $t$ steps is rather low and given by $N(1-\Phi(-\mu / \sigma))\left(1-(1-p)^{t}\right)$.
} 


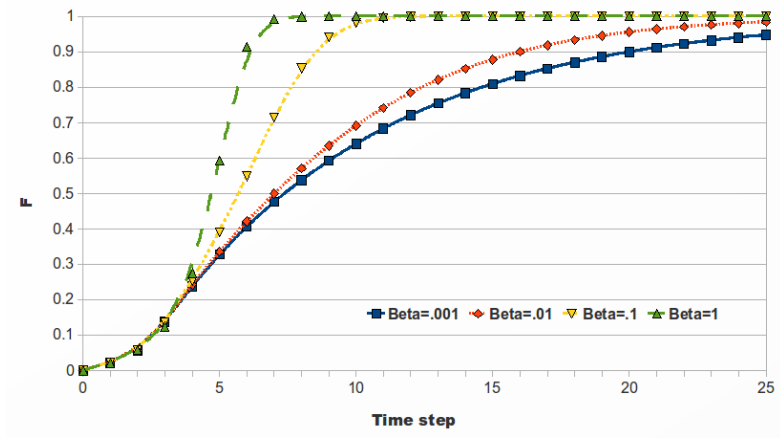

(a) Perfect information

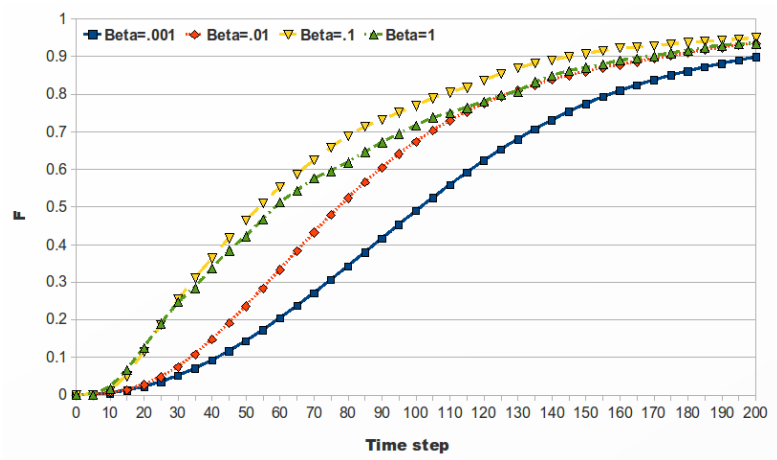

(b) Imperfect information

Fig. 2 Average cumulative frequency of adopters

decrease of clustering strongly reduces the possible reinforcement mechanisms in the initial steps of propagations. These mechanisms can be quite important in the early stages to make some agent adopt. Indeed, at the beginning, with imperfect information, propagation is triggered by very few adopters and the diffusion of information via demonstration effect, which requires previous adoption, is much stronger than the diffusion via broadcasting. ${ }^{13}$ Therefore, the increase of speed due to the slightly lower distance obtained by increasing further $\beta$ is not enough to compensate the decrease of speed produced by the lower clustering.

These results are confirmed in Figure 2, which shows the cumulative frequencies of adopters for different values of $\beta$ in the two regimes. In both cases, the four distributions - corresponding to four different values of $\beta$ - can be ordered in terms of first order stochastic dominance. However, with perfect information (Figure 2(a)) the random network $(\beta=1)$ is stochastically dominated by the others-i.e., it is unambiguously the most effective in diffusing promptly the innovation-, whereas with imperfect information (Figure $2(\mathrm{~b}))$ the small-world network $(\beta=0.1)$ is the dominated one.

\footnotetext{
13 In the simulation experiment, the information diffusion via demonstration effect is ten times more effective than via broadcasting: each new adopter can spread on average the information to 10 agents, whereas only 1 agent is on average informed via broadcasting at the same time.
} 


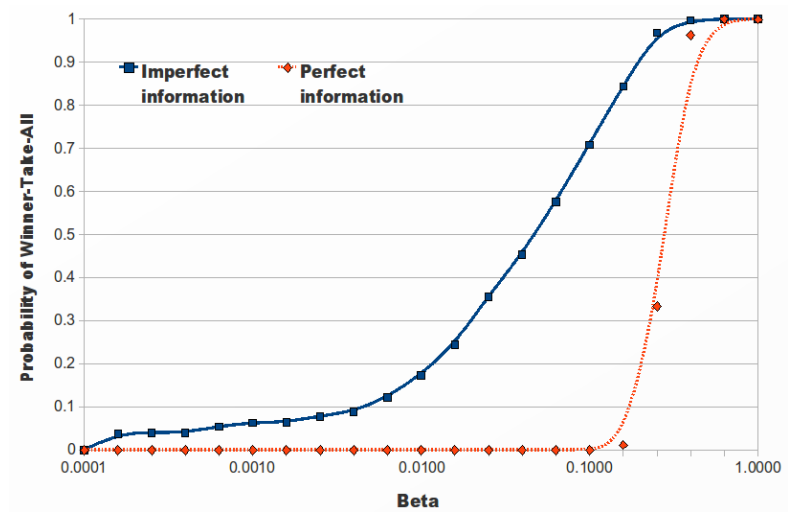

Fig. 3 Average probability of winner-take-all solution with perfect and imperfect information

Summing up, with perfect information, where all the agents are informed about the existence of the innovations and they only differ in their basic willingness-topay, it is important to reach quickly as many potential adopters as possible. In this case, with a rough tree-like structure, the random network better serves the purpose. On the contrary, with imperfect information, the small-world network is the most effective in the propagation process, since it provides the best interplay between the shortest way to communicate with the other people (low average distance) and the presence of reinforcing mechanisms in social influence (high clustering coefficient).

\subsection{Coexistence probability of competing innovations}

In what follows, we assume many incompatible product innovations competing in the market to analyze how the interplay between network structure and information regime can affect the probability of coexistence of alternative innovations. In so doing, we extend the results of the literature (e.g. Arthur 1989; Goyal and Janssen 1997; Dalle 1997; Cowan and Miller 1998; Lee et al. 2006) and gain new insights into the dynamics of competition when both network structure and information are taken into account.

In particular, we analyze the results of simulations for the case of five incompatible innovations, with perfect and imperfect information.

Figure 3 plots the probability of winner-take-all outcomes, defined as the share of simulation runs in which one innovation tips the market, against the usual parameter referring to the network structure. ${ }^{14}$ Data show that, with perfect information, when the clustering coefficient is high, there is always a solution with coexistence: because of the importance of social influence, cohesive groups of adopting agents shield against the alternative innovations. This result replicates and extends the one by Lee et al. (2006), who consider only two competing products.

14 The system is observed after 500 steps. The market shares stabilize after 30 and 200 steps in the perfect and imperfect information case respectively. 


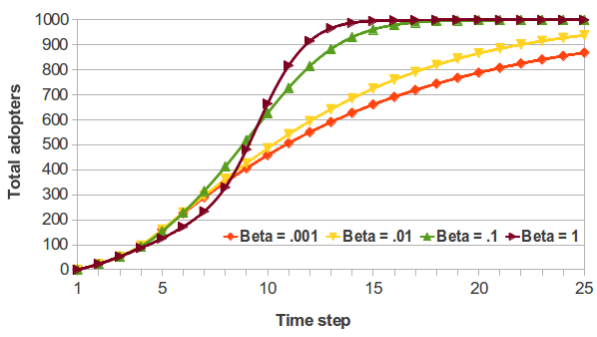

(a) Perfect information

Fig. 4 Average total number of adopters

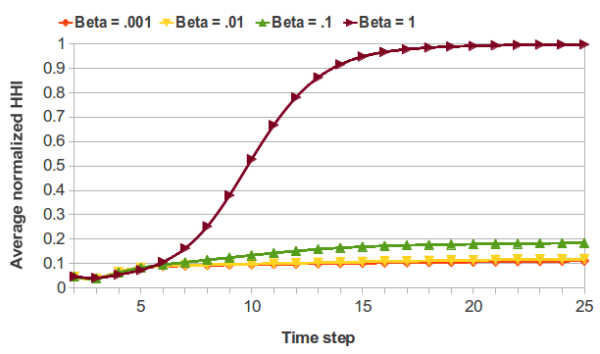

(a) Perfect information

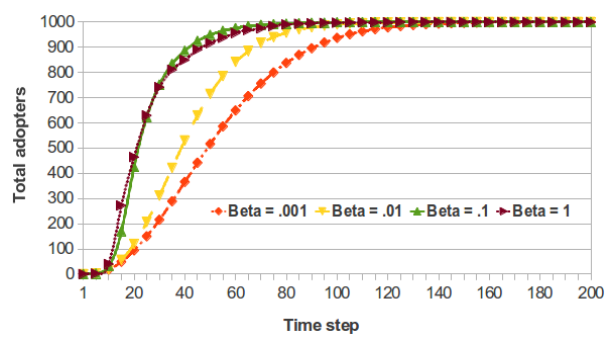

(b) Imperfect information

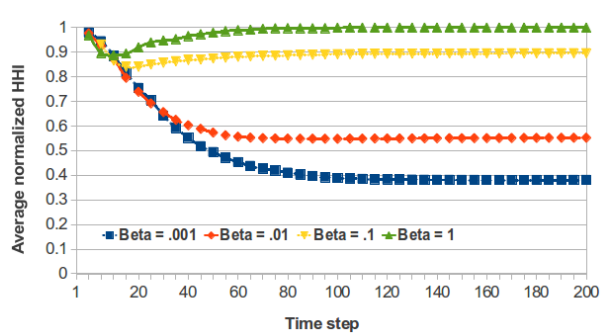

(b) Imperfect information

Fig. 5 Average normalized Herfindahl-Hirschman Index

However, Figure 3 also shows that such an outcome actually depends on the assumption of perfect information. Indeed, with imperfect information the effect of network configurations with a high clustering is significantly reduced. This occurs in particular when, despite the high clustering, the average distance is low, since this increases the speed of information diffusion via demonstration effect, and this in turn reduces the probability of formation of cohesive subgroups of adopters for the other innovations. ${ }^{15}$

Figures 4 and 5 clarify the underlying dynamics. Figure 4 shows the evolution of the average number of total adopters (of any innovation) with perfect and imperfect information. Figure 5 reports the dynamics of the average normalized Herfindahl-Hirschman Index (HHI), a measure of market concentration. ${ }^{16}$

15 The market shares are on average the same for all the innovations and equal to $20 \%$, because, apart from the external influence, each agent is assumed to be indifferent among them. Moreover, we find similar results for winner-take-all probabilities and market shares distributions by letting the idiosyncratic component of the consumer surplus $\left(r_{i}\right)$ vary for each individual with respect to each innovation $\left(r_{i x}\right)$ with values drawn from the same distribution.

16 The normalized HHI is equal to:

$$
\overline{\mathrm{HHI}}=\frac{\sum_{i=1}^{I} m_{i}^{2}-1 / I}{1-1 / I}
$$

where $m_{i}$ is the market share of product $i$ and $I$ the total number of products in the market. The index ranges from 0 to 1 and increases when the market concentration gets higher. 
The evolution of the average number of total adopters in the multi-product case are quite similar to that of the one-product case (Figure 2). However, the interesting aspect is the difference in the evolution of the average market concentration in the two regimes. With perfect information the initial concentration is close to zero and it then raises - and in random networks one product finally gets the whole market (Figure 5(a)). On the contrary, with imperfect information, at the beginning the average concentration in the market is high and it then decreases, when the other competing innovations start being adopted. Differences among the alternative network structures appear only after the 10th time step: in (quasi) regular networks HHI keeps on decreasing and then stabilizes, while in random networks, and somehow in small-world networks, it eventually increases (Figure $5(\mathrm{~b}))$.

The initial significantly higher market concentration can explain why winnertake-all solutions are on average higher in case of imperfect information across all the network configurations. However, it cannot account for the different results obtained for the different network structures in the same regime, which instead depend on the interplay between the information diffusion process and the characteristics of the network in terms of clustering and distance. This is confirmed by looking at the effects of different initial market shares on the probability of winner-tale-all solutions in case of perfect information. Indeed, confirming the findings of Lee et al. (2006, Section 4.2, p.1844), we find that with perfect information an initial high market concentration significantly increases the probability of winner-tale-all solutions only when the clustering coefficient starts falling $(\beta>0.1)$, contrary to what happens in case of imperfect information, where the probability starts getting bigger when the network becomes a small-world $(\beta>0.01)$.

Summing up, Lee et al.'s (2006) result on the low probability of winner-take-all in networks characterized by high clustering strongly depends on the assumption of perfect information. ${ }^{17}$ By relaxing this assumption, the winner-take-all probability is always positive and significantly higher. What is more, such probability sharply increases in networks characterized by low average distance, despite the possible high clustering.

Finally, it is worth noting that the assumption of imperfect information can be easily interpreted as one of lead-lag time in the introduction of the innovations in the market. Indeed, by assuming that not all agents are aware of the existence of all the innovations, we implicitly assume that one of them, i.e., the one adopted by the very first agent, is actually the first innovation introduced in the market and possesses therefore a lead-time advantage. All the other innovations face a disadvantage due to the experienced lag time.

4.3 Sensitivity analysis: agents' heterogeneity, strength of network externalities and penetration rate of innovations

For the sensitivity analysis of the model with one innovation and perfect information, we refer to Choi et al. (2010), who use the same model of Lee et al. (2006) with

\footnotetext{
17 Although Lee et al. (2006) are aware that "customer's information can sometimes be biased" (p.1843), the local bias they introduce cannot be properly considered an information bias, being related to other aspects of the social interaction/influence between the customer and her neighbors, namely emulation, fashion, seek for exchange standards, etc.
} 
only one product and carry out an extensive analysis. In particular, they show that lowering the portion of early adopters - by decreasing the expected value of $r_{i}(\mu)$ or its standard deviation $(\sigma)$-increases the probability of under-adoption, with the product not being adopted by more than $84 \%$ of the population. The highest increase occurs in random networks, that therefore are the least effective in diffusing innovation when the initial mass of adopters is small. The same effect is produced by weaker local network effects (lower $\alpha$ ).

The results we obtain for the case of imperfect information in the one-innovation case are similar: lowering the portion of potential early adopters (e.g. by decreasing $\mu)$ : i) lowers the speed of adoption; ii) increases the probability of falling into a trap of under-adoption. The latter effect is the highest when the network is random $(0.1<\beta \leq 1)$.

The results of the sensitivity analysis in the multi-innovation case are summarized in Table 1. We consider six alternative scenarios for each of the two information regimes: A) higher average basic willingness-to-adopt: $(\mu, \sigma)=(0,50)$; B) strictly positive willingness-to-adopt: $r>0$; C) higher heterogeneity: $(\mu, \sigma)=$ $(-100,200)$; D) higher heterogeneity and lower average willingness-to-adopt: $(\mu, \sigma)=(-200,100)$; E) weaker local network effects: $\alpha=250$; F $)$ weaker local network effects and higher heterogeneity: $\alpha=250$ and $(\mu, \sigma)=(-100,200)$. For each scenario we compute the total number of adopters and the normalized HHI after 500 time steps averaged across 1000 simulations. ${ }^{18}$

In scenario $\mathrm{A}$, the results are similar to the ones of the baseline scenario discussed in Section 4.2. All the agents end up adopting an innovation. With perfect information $(p=1)$, increasing $\beta$ leads to a significant raise in the expected concentration of the market only when the clustering collapses $(\beta>0.1)$; on the contrary, with imperfect information $(p=0.001)$, the average concentration in the market gets bigger for $\beta \geq 0.01$.

The same results hold in scenario $\mathrm{B}$, where a positive (and constant) basic willingness-to-adopt is assumed. ${ }^{19}$ In order to better grasp the relations between the different settings, it is worth stressing that, with this assumption and perfect information, each agent adopts immediately one innovation by choosing among them uniformly at random. She then revises her choice following a simple local majority rule. In this case, the analysis of the conditions for a winner-take-all solution to emerge resembles closely the analysis of the conditions for a local majority process to lead toward a consensus on the initial majority state. But, when a consensus is reached following a local majority rule, it is always on the initial majority state. So, the only issue in these analysis is when such rule actually leads to consensus. This is why, in the case of only two products and agents in a one-dimensional ring lattice, our analysis becomes formally equivalent to the density classification in one-dimensional binary-state cellular automata (Mitchell et al. 1994; Crutchfield and Mitchell 1995). In this setting, it has been proved that a local majority rule hardly leads to a consensus in the lattice, but the probability

\footnotetext{
18 Table 1 reports also for each scenario the expected fraction of early adopters $-\operatorname{Pr}(r \geq 0)-$ and the expected fraction of agents who will never adopt in any case $-\operatorname{Pr}(r \leq-\alpha)$.

19 The fact that $r$ is constant does not really matter. As far as $r$ is positive, its actual value does not affect the results, since it is equal for each agent across the alternative innovations.
} 
Table 1 Sensitivity analysis

\begin{tabular}{|c|c|c|c|c|c|c|c|c|c|c|c|}
\hline & \multicolumn{5}{|c|}{ Parameter values } & \multirow[b]{2}{*}{$\operatorname{Pr}(r \geq 0)$} & \multirow[b]{2}{*}{$\operatorname{Pr}(r \leq-\alpha)$} & \multicolumn{2}{|c|}{ Tot adopters } & \multicolumn{2}{|c|}{$\overline{\mathrm{HHI}}$} \\
\hline & $\mu$ & $\sigma$ & $\alpha$ & $p$ & $\beta$ & & & Avg & StDev & Avg & StDev \\
\hline \multirow[t]{4}{*}{ A1 } & 0 & 50 & 500 & 1.000 & 0.001 & 0.500 & 0.000 & 1000 & 0.03 & 0.02 & 0.01 \\
\hline & 0 & 50 & 500 & 1.000 & 0.010 & 0.500 & 0.000 & 1000 & 0.03 & 0.02 & 0.01 \\
\hline & 0 & 50 & 500 & 1.000 & 0.100 & 0.500 & 0.000 & 1000 & 0.06 & 0.03 & 0.03 \\
\hline & 0 & 50 & 500 & 1.000 & 1.000 & 0.500 & 0.000 & 1000 & 0.00 & 1.00 & 0.05 \\
\hline \multirow[t]{4}{*}{ A2 } & 0 & 50 & 500 & 0.001 & 0.001 & 0.500 & 0.000 & 1000 & 0.03 & 0.09 & 0.06 \\
\hline & 0 & 50 & 500 & 0.001 & 0.010 & 0.500 & 0.000 & 1000 & 0.00 & 0.15 & 0.10 \\
\hline & 0 & 50 & 500 & 0.001 & 0.100 & 0.500 & 0.000 & 1000 & 0.04 & 0.43 & 0.27 \\
\hline & 0 & 50 & 500 & 0.001 & 1.000 & 0.500 & 0.000 & 1000 & 0.00 & 1.00 & 0.00 \\
\hline \multirow[t]{4}{*}{ B1 } & 1 & 0 & 500 & 1.000 & 0.001 & 1.000 & 0.000 & 1000 & 0.00 & 0.02 & 0.01 \\
\hline & 1 & 0 & 500 & 1.000 & 0.010 & 1.000 & 0.000 & 1000 & 0.00 & 0.02 & 0.01 \\
\hline & 1 & 0 & 500 & 1.000 & 0.100 & 1.000 & 0.000 & 1000 & 0.00 & 0.03 & 0.02 \\
\hline & 1 & 0 & 500 & 1.000 & 1.000 & 1.000 & 0.000 & 1000 & 0.00 & 0.99 & 0.07 \\
\hline \multirow[t]{4}{*}{ B2 } & 1 & 0 & 500 & 0.001 & 0.001 & 1.000 & 0.000 & 1000 & 0.00 & 0.06 & 0.05 \\
\hline & 1 & 0 & 500 & 0.001 & 0.010 & 1.000 & 0.000 & 1000 & 0.00 & 0.10 & 0.07 \\
\hline & 1 & 0 & 500 & 0.001 & 0.100 & 1.000 & 0.000 & 1000 & 0.00 & 0.26 & 0.20 \\
\hline & 1 & 0 & 500 & 0.001 & 1.000 & 1.000 & 0.000 & 1000 & 0.00 & 1.00 & 0.00 \\
\hline \multirow[t]{4}{*}{$\mathrm{C} 1$} & -100 & 200 & 500 & 1.000 & 0.001 & 0.309 & 0.023 & 865 & 18.22 & 0.02 & 0.02 \\
\hline & -100 & 200 & 500 & 1.000 & 0.010 & 0.309 & 0.023 & 861 & 19.63 & 0.03 & 0.02 \\
\hline & -100 & 200 & 500 & 1.000 & 0.100 & 0.309 & 0.023 & 835 & 21.52 & 0.04 & 0.03 \\
\hline & -100 & 200 & 500 & 1.000 & 1.000 & 0.309 & 0.023 & 947 & 18.51 & 1.00 & 0.04 \\
\hline \multirow[t]{4}{*}{$\mathrm{C} 2$} & -100 & 200 & 500 & 0.001 & 0.001 & 0.309 & 0.023 & 907 & 19.79 & 0.08 & 0.05 \\
\hline & -100 & 200 & 500 & 0.001 & 0.010 & 0.309 & 0.023 & 900 & 20.03 & 0.13 & 0.09 \\
\hline & -100 & 200 & 500 & 0.001 & 0.100 & 0.309 & 0.023 & 885 & 31.24 & 0.38 & 0.23 \\
\hline & -100 & 200 & 500 & 0.001 & 1.000 & 0.309 & 0.023 & 948 & 12.08 & 1.00 & 0.02 \\
\hline \multirow[t]{4}{*}{ D1 } & -200 & 100 & 500 & 1.000 & 0.001 & 0.023 & 0.001 & 73 & 37.55 & 0.21 & 0.14 \\
\hline & -200 & 100 & 500 & 1.000 & 0.010 & 0.023 & 0.001 & 72 & 35.78 & 0.20 & 0.14 \\
\hline & -200 & 100 & 500 & 1.000 & 0.100 & 0.023 & 0.001 & 60 & 29.58 & 0.18 & 0.15 \\
\hline & -200 & 100 & 500 & 1.000 & 1.000 & 0.023 & 0.001 & 41 & 11.05 & 0.07 & 0.06 \\
\hline \multirow[t]{4}{*}{ D2 } & -200 & 100 & 500 & 0.001 & 0.001 & 0.023 & 0.001 & 865 & 18.17 & 0.02 & 0.02 \\
\hline & -200 & 100 & 500 & 0.001 & 0.010 & 0.023 & 0.001 & 861 & 19.64 & 0.03 & 0.02 \\
\hline & -200 & 100 & 500 & 0.001 & 0.100 & 0.023 & 0.001 & 835 & 21.49 & 0.04 & 0.03 \\
\hline & -200 & 100 & 500 & 0.001 & 1.000 & 0.023 & 0.001 & 947 & 18.47 & 1.00 & 0.04 \\
\hline \multirow[t]{4}{*}{ E1 } & -100 & 50 & 250 & 1.000 & 0.001 & 0.023 & 0.001 & 73 & 37.26 & 0.20 & 0.14 \\
\hline & -100 & 50 & 250 & 1.000 & 0.010 & 0.023 & 0.001 & 71 & 35.51 & 0.20 & 0.14 \\
\hline & -100 & 50 & 250 & 1.000 & 0.100 & 0.023 & 0.001 & 60 & 29.21 & 0.18 & 0.14 \\
\hline & -100 & 50 & 250 & 1.000 & 1.000 & 0.023 & 0.001 & 41 & 11.01 & 0.07 & 0.06 \\
\hline \multirow[t]{4}{*}{$\mathrm{E} 2$} & -100 & 50 & 250 & 0.001 & 0.001 & 0.023 & 0.001 & 84 & 43.92 & 0.28 & 0.17 \\
\hline & -100 & 50 & 250 & 0.001 & 0.010 & 0.023 & 0.001 & 80 & 42.98 & 0.28 & 0.17 \\
\hline & -100 & 50 & 250 & 0.001 & 0.100 & 0.023 & 0.001 & 58 & 31.68 & 0.27 & 0.17 \\
\hline & -100 & 50 & 250 & 0.001 & 1.000 & 0.023 & 0.001 & 29 & 10.13 & 0.16 & 0.12 \\
\hline \multirow[t]{4}{*}{ F1 } & -100 & 200 & 250 & 1.000 & 0.001 & 0.309 & 0.227 & 522 & 25.97 & 0.02 & 0.02 \\
\hline & -100 & 200 & 250 & 1.000 & 0.010 & 0.309 & 0.227 & 518 & 26.45 & 0.03 & 0.02 \\
\hline & -100 & 200 & 250 & 1.000 & 0.100 & 0.309 & 0.227 & 501 & 25.54 & 0.04 & 0.03 \\
\hline & -100 & 200 & 250 & 1.000 & 1.000 & 0.309 & 0.227 & 556 & 58.04 & 0.89 & 0.25 \\
\hline \multirow[t]{4}{*}{$\mathrm{F} 2$} & -100 & 200 & 250 & 0.001 & 0.001 & 0.309 & 0.227 & 537 & 27.54 & 0.05 & 0.04 \\
\hline & -100 & 200 & 250 & 0.001 & 0.010 & 0.309 & 0.227 & 532 & 26.81 & 0.08 & 0.06 \\
\hline & -100 & 200 & 250 & 0.001 & 0.100 & 0.309 & 0.227 & 519 & 28.60 & 0.30 & 0.19 \\
\hline & -100 & 200 & 250 & 0.001 & 1.000 & 0.309 & 0.227 & 578 & 29.57 & 1.00 & 0.04 \\
\hline
\end{tabular}

Total adopters and normalized Herfindahl-Hirschman Index $(\overline{\mathrm{HHI}})$ calculated after 500 time steps.

increases by increasing the number of shortcuts in the network and becomes very high when the network is nearly random (see, for instance, Watts 2003, Ch.7). ${ }^{20}$

In scenario C, a mean-preserving spread of the original distribution is assumed. Still, the main result on the relation between information regime, network structure and market concentration gets confirmed. Moreover, the increased variance with a constant mean also increases (decreases) the basic willingness-to-adopt for the

\footnotetext{
20 An analysis of a more general case with many different possible states is in Mustafa and Pekeč (2001), who show that, for any truly local network of agents, there are always instances in which the network is not capable of reaching the consensus following a local majority rule. On the more general decentralized consensus problem in multi-agent coordination, see also Ren et al. (2005) and Tahbaz-Salehi and Jadbabaie (2008).
} 
highest (lowest) percentiles of the population. Therefore, it is now simpler for an innovation to be adopted at the early stages of diffusion, but it is harder to eventually saturate the market. This explains, on the one side, the lower average number of total adopters with respect to the baseline scenario; on the other side, the higher number of total adopters in the case of imperfect information. Indeed, the coexistence among the alternative innovations necessarily decreases the consumer surplus and this in turn reduces the penetration. Because imperfect information implies an always higher average market concentration, this reduces the frictions due to the coexistence, increases the benefits of local network effects, hence the value of the innovation for the agents and this in turn increases the penetration rate. $^{21}$

These insights get confirmed in scenario D, where we assume a higher heterogeneity and a lower average willingness-to-adopt with respect to the baseline scenario. $^{22}$ The effects we have just described are here amplified and a striking difference emerges between the average total number of adopters in the two information regimes.

Under-adoption characterizes the outcomes of scenario E, where we assume weaker local network effects, the only driver of diffusion when the basic willingnessto-adopt is negative (less than $8 \%$ of the population eventually adopt an innovation on average). Also in this case the coexistence of alternative innovations can hurt diffusion and a strong cliquishness is needed to sustain it and to avoid falling into a trap of under-adoption. Like in Choi et al. (2010), such probability is maximal for a random network.

Finally, in scenario $\mathrm{F}$ we increase the heterogeneity in the population, still assuming weak network effects. This is enough to significantly increase adoption and escaping the trap of under-adoption (more than 50\% of the population adopt an innovation). The familiar relation between information regime, network structure and market concentration reappears.

Summing up, we confirm and generalize Choi et al.'s (2010) results on the relation between network structure and penetration rates for the case of more than one product: a weak cliquishness increases the probability of falling into a trap of under-adoption. More important, we find that such probability is significantly lower in case of imperfect information, cause this regime is associated with a higher initial market concentration, which increases the level of positive network externalities associated with the adoption, thus enhancing the penetration of the product.

\section{Concluding remarks}

The paper analyzes the role that the structure of interaction networks has on diffusion patterns and markets shares of different innovations with local network effects under different information regimes.

\footnotetext{
21 Let us note in passing that in our model, consumer surplus is maximized in the case of a winner-take-all outcome, and that therefore coexistence is always not optimal from a social point of view. This result clearly depends on the fact that we are neglecting the supply-side effects of competition.

22 The values are such that the coefficient of variation of $r$ is the same of the original distribution.
} 
We put forward a theoretical model where the diffusion of different innovations in the market is modeled as the result of two (only partly) interrelated dynamics: i) the interaction between idiosyncratic adoption thresholds and local network effects; ii) the diffusion of information on the innovation. As for the latter, we differentiate between a situation of: a) perfect information, where customers are perfectly informed about the existence of the different innovations and can freely choose among them; b) imperfect information, where not all potential customers are informed about innovations' availability at each moment in time and this information diffuses through the network via broadcasting and demonstration effect from previous adopters.

We implement the model as an agent-based model and discuss the results of the simulations for the one-innovation and many-innovations case. As for the former, with perfect information, the random network with its quasi tree-like structure is the one that allows to saturate the market first, whereas instead, with imperfect information, the small-world network is the most efficient in glutting the market, because of its high clustering coupled with a low average distance.

In the case of many innovations competing for the same market, we analyze the probability of one of them displacing completely the others and cornering the market. We are thus able to show that, in a situation of perfect information, what really matters is just the level of clustering: as far as it is above a certain level, there is always an outcome with coexistence and no standardization. On the contrary, under imperfect information, the importance of network configurations with a high clustering is significantly reduced, yielding an increased winner-take-all probability. And such probability increases steadily with the decrease of the average distance in the network.

Our results actually show that, in case of networks characterized by high clustering and low average distance, as the social ones, with imperfect information and low marketing efforts to launch the different innovations, the probability of an outcome with coexistence is rather low. Moreover, such an outcome is much less probable when there are even small time lags in the introduction of the different innovations. And this can partly explain why marketing efforts of firms are usually quite high: to dump the reinforcement mechanisms behind adoption of innovation and information diffusion processes for the competitors when the information is not perfect.

Moreover, we confirm and generalize Choi et al.'s (2010) results on the relation between network structure and penetration rates for the case of more than one product: a weak cliquishness increases the probability of falling into a trap of under-adoption. Moreover, we find that such probability is significantly lower in case of imperfect information, cause this regime is associated with a higher initial market concentration, which increases the level of positive network externalities associated with the adoption, thus significantly enhancing the penetration of the product in the first stages.

Our findings seem interesting in three respects. First of all, the model developed in the paper puts together different streams of literature dealing with innovation diffusion. In particular, it combines the two main classes of models: threshold (or probit) models and epidemic ones. The former class properly accounts for the factors affecting adoption decisions, but it makes a restrictive assumption of perfect information. The latter properly models imperfect information, but it also assumes that, when agents get in touch with the innovation, they eventually end 
up adopting. Ours is instead a mixed model of diffusion (Geroski 2000), that puts together the epidemic character of information diffusion with the threshold and idiosyncratic nature of the adoption decision, which is nonetheless affected by local network effects.

Second, we extend and generalize the results about the diffusion of innovation of a single innovation in markets characterized by fads and fashions by stressing the inner dynamics of the phenomenon. In particular, we show that by relaxing the assumption of perfect information, the efficiency ranking of network structures in terms of diffusion changes.

Third, we extend the results of the literature about the competition among multiple innovations by taking into account more than two innovations and allowing the presence of lead-lag time. In so doing, we are able to show how the results of the previous literature strongly depend on the assumption of perfect information (that can be also interpreted as a hypothesis of absence of lead-lag times in the introduction of innovations in the market).

The model has straightforward application in fashionable markets, where social processes such as imitation, status seeking, peer and demonstration effects are essential; but also in markets where the relational aspects constitute a strong part of the value attached to the product. Noteworthy examples are the markets for technological standards, electronic devices, software and Social Networking Sites (SNS).

SNSs are a particularly interesting example. They are popular communication interfaces for hundreds of millions of users. In the last years, social networks have attracted a large share of the population. Facebook, with its over 600 million members worldwide, is the most well-known case and has recently surpassed Google as the most visited website in the US. Although it was not the first social network entering the market-i.e., other social networking sites such as Friendster, MySpace and CyWorld were well before Facebook-, it has been able to become in a few years the absolute leader (Boyd and Ellison 2008). Unlike previous SNSs, Facebook went through a number of steps before opening to everyone. At the beginning, it was designed to support distinct college networks only, after some time it expanded to include high school students, professionals inside corporate networks, and, eventually, everyone. Furthermore, unlike other SNSs, Facebook users are unable to make their full profiles public to all users. Another feature that differentiates Facebook is the ability for outside developers to build "applications" which allow users to personalize their profiles and perform other tasks (e.g. compare movie preferences and chart travel histories). All these features helped Facebook to build its own installed base and, by exploiting local network effects and information diffusion, to mostly corner the market in a setting characterized by hardly perfect information and a high clustering but still very low distance in the structure of social interactions ( $\beta$ roughly 0.1 ).

Another real world example is provided by the Voice over Internet Protocol (VoIP) technology. The use of Internet for voice communications has been an important innovation for the telecommunication and the Internet industry. These sectors have been characterized by fierce competition in recent years. Although extensive data on market shares of companies operating in the VoIP market segment are lacking, a recent survey of VoIP end users by Cecere and Corrocher (2011) provides some figures for the UK market. As it was largely expected, Skype is the most popular provider with $67 \%$ of the market share, MSN is the most popular 
operator (18\% of users) and BT/Yahoo! is the third (16\%). It is evident from these numbers that Skype is the leader in the market and that, with the recent acquisition by Microsoft, it will reach the $85 \%$ of the user base. VoIP innovation is thus a case where the exploitation of first-mover advantage and strong local network effects present in the market allowed one company to almost tip the market.

These examples give us also the opportunity to point out some factors that might play a major role in reality and we have instead deliberately neglected in our model. First, the role of economies of scale on the production side, which tend to increase the probability of winner-take-all outcomes. Second, the possibility of joint adoption of alternative innovations and the presence of switching costs (stemming from processes of learning-by-using and sunk costs connected with the adoption), which instead raise the likelihood of outcomes with coexistence.

Moreover, we have deliberately abstracted from the supply-side effects of competition. In fact, firms may compete on process innovation and the diffusion observed in many goods may be the result of changes in price, rather than imitation and/or network externalities. This could also open up a discussion on conspicuous consumption, income and social relations, which can be considered for future research. $^{23}$

Finally, an important factor to consider is the high heterogeneity in the number of links of each agent in social networks. Indeed, in some social networks (friendship networks in SNSs are a notable example), node degree follows a power-law distribution that becomes truncated or exponential only at very high values (Newman 2003). ${ }^{24}$ If the resulting network were the scale-free network of Albert and Barabási (2002), the probability of coexistence would be strongly reduced (Uchida and Shirayama 2008). However, one should consider other countervailing forces, such as a possible high clustering and/or assortativity (degree correlation). ${ }^{25}$ As a matter of fact, this is a topic not yet completely explored and that deserves more research.

Acknowledgements Francesco Rentocchini and Giuseppe Vittucci Marzetti gratefully acknowledge financial support from the Autonomous Province of Trento (Grants 2006: TRACKs and FIC). The authors thank Marco Valente for the "after-sale support" of LSD. They also acknowledge the participants to the Workshop on "Structure and Dynamics of Knowledge Networks" (Eindhoven, May 12-14, 2009) and two anonymous referees for very useful and detailed comments. The usual caveats apply.

\section{References}

Abrahamson, E, and L Rosenkopf. 1997. Social network effects on the extent of innovation diffusion: A computer simulation. Organization Science 8 (3): 289-309.

23 We acknowledge one of the referee for this suggestion.

24 What is more, some of these power law distributions has an exponent equal or less than 3 , and hence no finite variance. Sometimes they even exhibit an exponent equal or less than 2 , and therefore they have no finite mean either (Newman 2005).

25 These higher-order properties do not automatically descend from local (first-order) properties of the network, like the degree sequence. In fact, Bollobás and Riordan (2003) show that Albert and Barabási's (2002) model is not even well-defined, since it is consistent with several models that are different with respect to their higher-order properties. 
Albert, R, and AL Barabási. 2002. Statistical mechanics of complex networks. Reviews of Modern Physics 74: 47-97.

Arenas, A, A Díaz-Guilera, CJ Perez, and F Vega-Redondo. 2002. Self-organized criticality in evolutionary systems with local interaction. Journal of Economic Dynamics and Control 26 (12): 2115-2142.

Arthur, BW. 1989. Competing technologies, increasing returns, and lock-in by historical events. The Economic Journal 99 (394): 116-131.

Banerji, A, and B Dutta. 2009. Local network externalities and market segmentation. International Journal of Industrial Organization 27 (5): 605-614.

Bass, FM. 1969. A new product growth for model consumer durables. Management Science 15: $215-227$.

Bass, FM. 2004. Comments on a new product growth for model consumer durables. the Bass model. Management science.

Bemmaor, AC, and J Lee. 2002. The impact of heterogeneity and ill-conditioning on diffusion model parameter estimates. Marketing Science 21 (2): 209-220.

Bollobás, B, and OM Riordan. 2003. Mathematical results on scale-free random graphs. In Handbook of graphs and networks. from the genome to the internet, eds. S. Bornholdt and HG Schuster, 1-34. Weinheim: Wiley-VCH.

Boyd, DM, and NB Ellison. 2008. Social network sites: Definition, history, and scholarship. Journal of Computer-Mediated Communication 13 (1): 210-230.

Bramoullé, Y, and R Kranton. 2007. Risk sharing across communities. American Economic Review 97 (2): 70-74.

Cecere, G, and N Corrocher. 2011. The intensity of VoIP usage in Great Britain: Users' characteristics and firms' strategies. Telecommunications Policy 35 (6): 522-531.

Chatterjee, R, and J Eliashberg. 1990. The innovation diffusion process in a heterogeneous population: A micromodeling approach. Management Science 36 (9): 1057-1079.

Choi, H, S-H Kim, and J Lee. 2010. Role of network structure and network effects in diffusion of innovations. Industrial Marketing Management 39 (1): 170-177.

Cowan, R, and N Jonard. 2003. The dynamics of collective invention. Journal of Economic Behavior \& Organization 52 (4): 513-532.

Cowan, R, and N Jonard. 2004. Network structure and the diffusion of knowledge. Journal of Economic Dynamics and Control 28 (8): 1557-1575.

Cowan, R, and JH Miller. 1998. Technological standards with local externalities and decentralized behaviour. Journal of Evolutionary Economics 8 (3): 285-296.

Crutchfield, JP, and M Mitchell. 1995. The evolution of emergent computation. Proceedings of the National Academy of Sciences 92 (23): 10742-10746.

Dalle, JM. 1997. Heterogeneity vs. externalities in technological competition: A tale of possible technological landscapes. Journal of Evolutionary Economics 7 (4): 395-413.

Delre, SA, W Jager, and MA Janssen. 2007a. Diffusion dynamics in small-world networks with heterogeneous consumers. Computational \& Mathematical Organization Theory 13 (2): $185-202$.

Delre, SA, W Jager, THA Bijmolt, and MA Janssen. 2007b. Targeting and timing promotional activities: an agent-based model for the takeoff of new products. Journal of business research 60 (8): 826-835.

Delre, SA, W Jager, THA Bijmolt, and MA Janssen. 2010. Will it spread or not? The effects of social influences and network topology on innovation diffusion. Journal of Product Innovation and Management.

Frenken, K, G Silverberg, and M Valente. 2008. A percolation model of the product life cycle, Working Paper Series 73, UNU-Merit.

Geroski, PA. 2000. Models of technology diffusion. Research Policy 29 (4-5): 603-625.

Golub, B, and MO Jackson. 2010. Naïve learning in social networks: Convergence, influence, and the wisdom of crowds. American Economic Journal: Microeconomics 2 (1): 112-149.

Goyal, S. 2007. Connections: An introduction to the economics of networks. Princeton: Princeton University Press.

Goyal, S, and MCW Janssen. 1997. Non-exclusive conventions and social coordination. Journal of Economic Theory 77 (1): 34-57.

Goyal, S, and JL Moraga-Gonzalez. 2001. R\&D networks. The RAND Journal of Economics 32 (4): $686-707$.

Hohnisch, M, S Pittnauer, and D Stauffer. 2008. A percolation-based model explaining delayed takeoff in new-product diffusion. Industrial and Corporate Change 17 (5): 1001-1017. 
Jackson, MO. 2008. Social and economic networks. Princeton: Princeton University Press.

Janssen, MA, and W Jager. 2003. Simulating market dynamics: Interactions between consumer psychology and social networks. Artificial Life 9 (4): 343-356.

Karshenas, M, and P Stoneman. 1995. Technological diffusion. In Handbook of the economics of innovation and technical change, ed. P Stoneman. Basil Blackwell.

Katz, ML, and C Shapiro. 1985. Network externalities, competition, and compatibility. American Economic Review 75 (3): 424-440.

Krishnamurthy, S. 2003. A managerial overview of open source software. Business Horizons 46 (5): $47-56$.

Lee, E, J Lee, and J Lee. 2006. Reconsideration of the winner-take-all hypothesis: Complex networks and local bias. Management Science 52 (12): 1838-1848.

López-Pintado, D. 2008. Diffusion in complex social networks. Games and Economic Behavior 62 (2): 573-590.

Mahdian, M, J Kleinberg, N Immorlica, and T Wexler. 2007. The role of compatibility in the diffusion of technologies through social networks. In Proc. 8th acm conference on electronic commerce.

Mitchell, M, JP Crutchfield, and PT Hraber. 1994. Evolving cellular automata to perform computations: mechanisms and impediments. Physica D: Nonlinear Phenomena 75 (1-3): 361-391.

Morris, S. 2000. Contagion. Review of Economic Studies 67 (1): 57-78.

Morrison, A. 2008. Gatekeepers of knowledge within industrial districts: who they are how they interact. Regional Studies 42: 817-835.

Mustafa, N, and A Pekeč. 2001. Majority consensus and the local majority rule. In Automata, languages and programming, eds. F Orejas, P Spirakis, and J van Leeuwen. Vol. 2076 of Lecture notes in computer science, 530-542. Berlin/Heidelberg: Springer.

Newman, MEJ. 2003. The structure and function of complex networks. SIAM Review 45: $167-256$.

Newman, MEJ. 2005. Power laws, Pareto distributions, and Zipf's law. Complexity Digest 02: $1-27$.

Ren, W, RW Beard, and EM Atkins. 2005. A survey of consensus problems in multi-agent coordination. In American control conference, 2005. proceedings of the 2005, Vol. 3, 18591864.

Rogers, EM. 2003. Diffusion of innovation. New York: Free Press.

Swann, GM. 2002. The functional form of network effects. Information Economics and Policy 14 (3): 417-429.

Tahbaz-Salehi, A, and A Jadbabaie. 2008. A necessary and sufficient condition for consensus over random networks. IEEE Transactions on Automatic Control 53 (3): 791-795.

Uchida, M, and S Shirayama. 2008. Influence of a network structure on the network effect in the communication service market. Physica A: Statistical Mechanics and its Applications 387 (21): 5303-5310.

Uzzi, B, LAN Amaral, and F Reed-Tsochas. 2007. Small-world networks and management science research: a review. European Management Review 4 (2): 77-91.

Valente, TW. 2005. Network models and methods for studying the diffusion of innovation. In Models and methods in social network analysis, eds. PJ Carrington, J Scott, and S Wasserman, 98-116. Cambridge: Cambridge University Press.

Vega-Redondo, F. 2007. Complex social networks. Cambridge: Cambridge University Press.

Watts, DJ. 2003. Small worlds: the dynamics of networks between order and randomness. Princeton: Princeton University Press.

Watts, DJ, and SH Strogatz. 1998. Collective dynamics of "small-world" networks. Nature 393: 440-442.

Young, HP. 2009. Innovation diffusion in heterogeneous populations: Contagion, social influence, and social learning. American Economic Review 99 (5): 1899-1924. 